\title{
Entités Territoriales Communales Décentralisées et Gouvernance des Ressources Foncières : L'exemple de l'or dans le Ganadougou/Région de Sikasso au Mali
}

\author{
Dr. Siaka Fane, \\ Faculté d'Histoire et Géographie (FHG) de l'Université des Sciences \\ Sociales et de Gestion de Bamako (USSGB), Géographe

\section{Dr. Issa Samake,} \\ Institut Universitaire de Technologie (IUT) de l'Université des Lettres et des \\ Sciences Humaines de Bamako (ULSHB), Economiste
}

Doi:10.19044/esj.2019.v15n29p117 URL:http://dx.doi.org/10.19044/esj.2019.v15n29p117

\section{Résumé}

Le Mali est un pays très riche en or. Les orientations économiques des politiques de développement ont contribué à mettre les ressources aurifères au cœur d'une ferveur considérable. Il en résulte des enjeux socio-économiques et politiques à l'œuvre dans la transformation des territoires au plan national et local. Du coup, la question de l'exploitation de l'or, quelle que soit sa forme, présente un intérêt scientifique évident pour les géographes et les économistes. La présente recherche vise certains objectifs. Il s'agit de connaitre l'importance et la nature des pratiques d'exploitation de l'or ; de connaitre les modalités de la gouvernance des ressources foncières aurifères ; de faire une analyse de la traduction spatiale du développement lié à la gouvernance en place. Leur atteinte a nécessité l'observation d'une démarche méthodologique. Celle-ci a été fondée sur l'exploitation de la documentation et les enquêtes de terrain. Fondamentalement les résultats ont permis de comprendre qu'à partir de l'orpaillage traditionnel dont ils assurent la gouvernance, les populations du Ganadougou ont remodelé leur espace en deux types distincts de territoires. Le premier est socialement et économiquement développé. Il s'oppose au deuxième très arriéré sur les mêmes plans.

Mots-clés: Orpaillage, Développement économique, Développement social, Villages sites d'orpaillage, Villages non sites d'orpaillage, Ganadougou 


\title{
The Decentralized, Communal, and Territorial Entities and the Governance of Land Resources: The Case of Gold in Ganadougou/ Area of Sikasso in Mali
}

\author{
Dr. Siaka Fane, \\ Faculté d'Histoire et Géographie (FHG) de l'Université des Sciences \\ Sociales et de Gestion de Bamako (USSGB), Géographe \\ Dr. Issa Samake, \\ Institut Universitaire de Technologie (IUT) de l'Université des Lettres et des \\ Sciences Humaines de Bamako (ULSHB), Economiste
}

\begin{abstract}
Mali is a country very rich in gold. The economic policy orientations have contributed to putting the gold resources in the middle of a considerable zeal. This leads to the active socio-economic stakes in the transformation of territories at both national and local level. Consequently, the gold operation issue, whatever its form is, presents an obvious scientific interest for the geographers as well as for the economists. This paper focuses on achieving the following goals: to know the importance and the nature of gold operation practices, to know the governance methods of gold property resources, and to analyze the space manifestation of development due to the setting up governance. A methodological approach was made to reach the goals of this research. It was based on documentation process and field surveys. Basically, the results have made it possible to understand that from traditional mining of which the peoples of Ganadougou ensure the governance, they reshaped their space in two separate types of territories. The first is socially and economically developed, which is opposed to the second that is more backward on the same plans.
\end{abstract}

Keywords: Placer mining, Economic development, Social development, Villages with placer mining sites, Villages without placer mining sites, Ganadougou 


\section{Introduction}

Le Mali figure dans le peloton des pays africains riches en or. Il occupe, après le Ghana, le Soudan, l'Afrique du sud, la $4^{\text {ème }}$ place continentale (WebNewsle360afrique.com). A la clef de l'honorable rang malien prévaut une importante activité d'exploitation de l'or. Elle est animée par deux secteurs. Le premier est industriel. Les mines qui y relèvent sont nombreuses. Leur multiplicité s'explique par le fait que depuis la fin des années 1990, les institutions financières internationales promeuvent l'initiative privée comme moteur du développement socio-économique (Thune, 2011).

Le second, très ancien, est artisanal. C'est dans son cadre que les mines d'or étaient, d'après El-Bakri (XIe siècle) et Al-Idrisi (XIIe siècle), sous le monopole du roi du Ghana (Panella, 2007). Ce secteur artisanal, pour des questions de sa typologie, a fait l'objet de débats. D'une façon générale, des critères sont toujours évoqués dans les tentatives de définition (Keita, 2001). En intégrant les différents critères de classification et le caractère traditionnel de l'exploitation minière à petite échelle, le code minier du Mali de 2012 s'appuie sur les considérations de simplicité des équipements utilisés et des technologies mises en œuvre d'une part, la faiblesse des investissements nécessaires à l'opération ainsi que la non indispensable grande qualification des travailleurs miniers, d'autre part. Sur cette base, les dispositions du code minier identifient deux grands types d'activités dans le concept de l'exploitation minière à petite échelle et les définissent comme suit : la petite mine et l'exploitation traditionnelle. Le dernier type, objet du présent article, s'adresse à toute activité qui consiste à récupérer, par des procédés artisanaux, des substances précieuses en l'occurrence l'or et le diamant. Cette activité reste organisée par la communauté villageoise. Quant au second il porte sur toute activité d'orpaillage mieux structurée que l'orpaillage traditionnel et qui fait appel à l'usage de certaines machines.

A l'échelle spécifique du Ganadougou des sites d'exploitation de l'or sont ouverts et fonctionnels dans plusieurs villages. Cet espace du Ganadougou a, suite à la loi n96-059 du 04 novembre 1996, fait l'objet de découpage entre dix communes rurales. Y aborder la gouvernance des ressources aurifères revient à inscrire la présente recherche dans la thématique de la gouvernance locale dont la clarification conceptuelle est, dans les études de beaucoup d'auteurs, fondée sur son objectif. Pour eux la gouvernance locale vise à transférer le pouvoir aux populations locales en vue de réaliser un développement économique et politique qui soit mené par les populations elles-mêmes et qui met l'accent sur la réduction de la pauvreté (Bonfigiali, 2003 cité par Iram, 2008). Une telle définition est source d'importantes confusions. En effet, à l'échelle des entités territoriales communales la décentralisation a introduit une superposition officielle de nouvelles structures 
de gouvernance sur des institutions coutumières existantes. Il s'agit du conseil municipal ayant à sa tête le maire. Il jouit de légitimité suivant les lois sur la décentralisation. Ces dernières énoncent les responsabilités des collectivités territoriales concernant l'utilisation et la gestion des ressources naturelles (Hilhorst, 2008).

A la lumière des différentes réalités, ci-dessus brossées, certains faits majeurs caractérisent les entités territoriales décentralisées du Ganadougou. Il s'agit de l'existence des pratiques d'exploitation de l'or et l'existence de différentes instances de gouvernance (coutumière, conseil municipal). Ce contexte fait naitre des interrogations. Elles sont relatives à la nature et à l'importance des pratiques d'exploitation ; à la forme de la gouvernance et au développement socioéconomique y inhérent.

\section{Matériel et Méthodes}

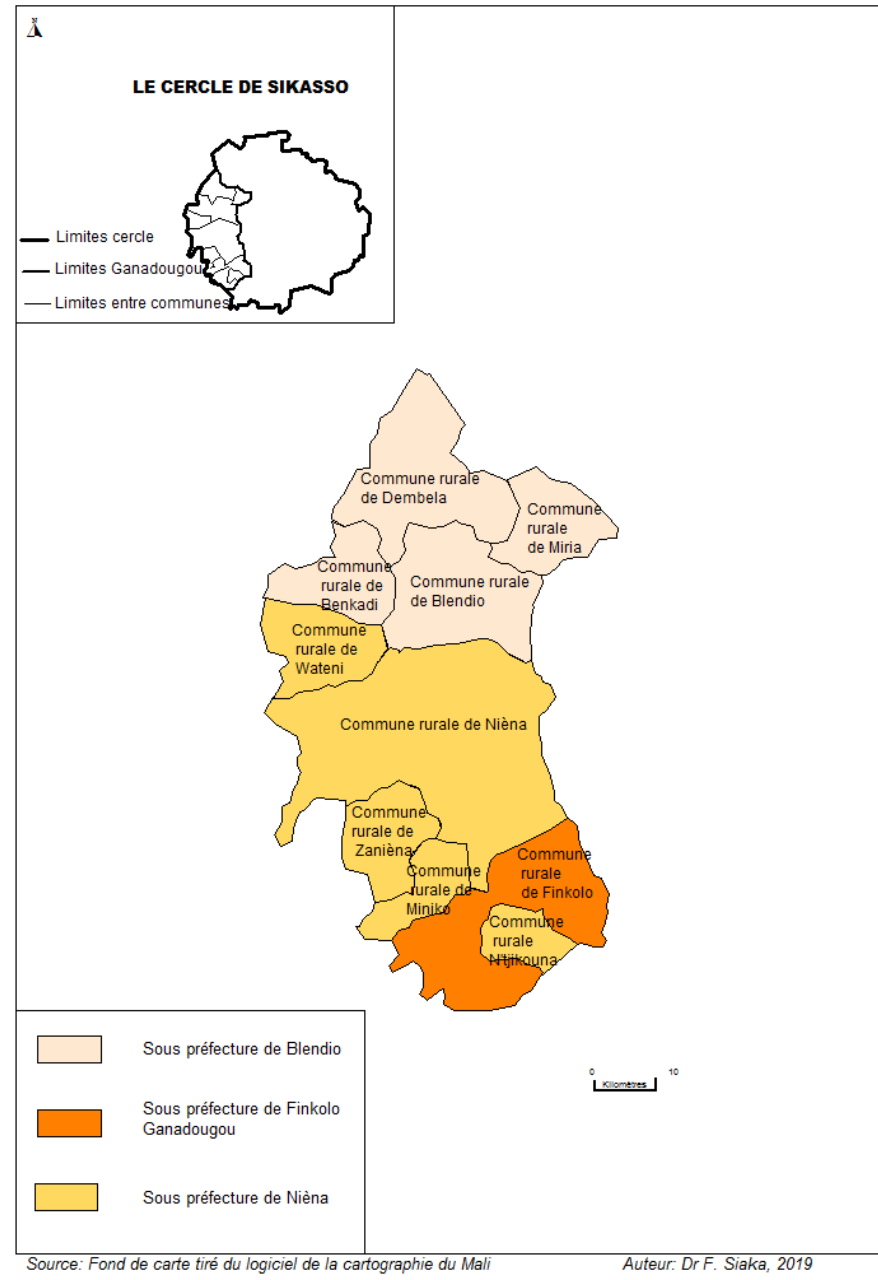

Carte $n^{\circ} 1$. Les communes rurales du Ganadougou par sous-préfecture 
Terme fédérateur, le Ganadougou est une entité spatiale bien individualisée par un dialecte de la langue bambara. Sa localisation

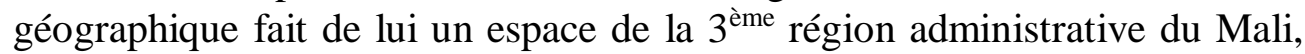
région de Sikasso. Il fait l'objet de découpage entre trois arrondissements ou sous-préfectures dits de Blendio, de Finkolo Ganadougou et de Nièna. Ces derniers sont différemment concernés par l'avènement de la décentralisation. Blendio et Nièna comptent respectivement cinq (5) et quatre (4) communes rurales et Finkolo Ganadougou une (1) commune rurale, voir carte $n^{\circ} 1$, cidessus.

Le climat est de type tropical sud soudanien. Il se caractérise, compte tenu de la moyenne du Mali, par une pluviométrie abondante de $800 \mathrm{~mm}$ à $1000 \mathrm{~mm}$ d'eau par an. La saison des pluies, longue de 5 à 6 mois (mai à octobre) avec plus de 90 jours de pluie par an, connait une saison sèche (novembre à avril). En saison des pluies le vent dominant est la mousson chargée d'humidité qui souffle du sud-ouest au nord-est et en saison sèche, c'est l'harmattan, vent chaud et sec venant du nord-est.

Le relief est en général plat et monotone, formé de champ de collines, de plaine et de plateau. La latitude moyenne de cet ensemble tabulaire se situe entre 300 et 400 mètres. C'est ainsi que des vastes plaines inondées s'étendent dans plusieurs villages du Ganadougou comme Fougani, Pankourou, etc. A celles-ci s'ajoutent de nombreux bas-fond dont certains ont fait l'objet d'aménagement. Les sols sont de type limono-argileux, limono-sablonneux, argileux et gravillonnaire.

$\mathrm{Au}$ plan végétatif nous avons la forêt claire et la savane qui caractérisent le mieux le cadre physique du Ganadougou. Dans les vallées, ce sont des galeries forestières. L'hydrographie est formée par plusieurs rivières permanentes disposant d'affluents localement désignés «kô». Ils sont temporaires. A ceux-ci s'ajoutent des marres plus ou moins vastes. La plus importante est celle de Kougnan dans la commune rurale de Benkadi.

Les différents éléments physiques, abordés supra, offrent aux populations les opportunités de pratiquer diverses activités. Ce sont la production agricole, l'élevage, la pêche, la production des bois et du charbon, l'extraction de l'or, le commerce, etc.

\section{Méthodes et outils}

La méthodologie observée comporte certains points. Le premier est la collecte des informations. Sa conduite s'est faite en deux temps.

- L'exploitation de la documentation a conduit à recourir à certains documents. Ils se rapportent à diverses thématiques.

Les enquêtes de terrain ont nécessité l'administration de questionnaire. A cet effet, un échantillonnage a été fait à deux niveaux.

1- Les villages ont fait l'objet de choix en deux temps. 
-Dans le premier temps les nécessités d'atteinte du premier et deuxième objectif ont conduit à considérer les villages sites d'orpaillage. Le Ganadougou compte 19 villages sites d'orpaillage. Ils ont été répartis en trois catégories. Ce sont les anciens sites (âge $\geq 10$ ans) ; les sites récents (âge $<10$ ans et $\geq 5$ ans) ; les sites très récents (âge $<5$ ans). Pour la présente recherche 6 villages sites d'orpaillage ont été retenus soit environ le 1/3 des 19. Ainsi, deux villages ont été tirés au hasard par catégorie de sites.

-Dans le deuxième temps les deux catégories de villages (site ou non site d'orpaillage) ont été considérées en rapport avec le troisième objectif. A cet effet, six (06) villages sites d'orpaillage et quatre (04) villages non sites d'orpaillage ont été aléatoirement retenus. Faisant un total de 10 villages, ils ont été tirés au hasard.

2- Les cibles d'enquête forment deux groupes

- Les autorités sont représentées par les maires et les chefs de villages. Les villages tirés et sites des enquêtes relèvent des communes de Nièna et de Finkolo Ganadougou. Ils sont également dirigés chacun par un chef de village. Ainsi, les maires et 6 chefs de villages ont été retenus.

- Les populations sont responsables de la pratique d'extraction de l'or. Cette dernière s'inscrit dans un jeu d'acteurs collectifs. Les enquêtes individuelles n’ont pas été jugées pertinentes. Aussi, des échanges ont-ils eu lieu sous les hangars construits sur les sites et en présence de plusieurs orpailleurs. Sans véritablement remplir les normes de nombre de personnes (30 au moins), le travail s'est effectué quasiment en focus groupe. En fonction des villages les questions ont été posées à ceux qui ont été disponibles. Au total six focus groupes ont été faits. Contrairement à la première modalité de focus groupe, des enquêtes individuelles ont été faites auprès des populations relativement aux préoccupations de développement. Lesdites enquêtes ont concerné un échantillon aléatoire de cent (100) personnes environ en raison de quarante (40) dans les villages non sites d'orpaillage et soixante (60) dans les villages sites d'orpaillage.

Le deuxième point méthodologique renvoie au traitement des informations. Celles collectées ont été automatiquement traitées au moyen des logiciels EXCEL et SPSS.

\section{Resultats et Discussion}

Le Ganadougou et la Pratique de l'exploitation des Ressources Auriferes

S'intéresser à la pratique d'une activité dans un espace conduit à aborder divers éléments. Ils ne relèvent pas d'un standard. C'est justement des résultantes des objectifs de chaque recherche. Aussi, deux éléments importentils dans le cadre de la présente recherche. 


\section{La situation des équipements utilisés aux fins de typologie de l'activité d'exploitation de l'or}

Conformément aux indications du code minier du Mali de 2012 les équipements constituent des éléments fondamentaux de détermination de la nature de l'activité d'exploitation aurifère. La situation de ceux détenus et utilisés par les exploitants est donnée dans le Tableau ${ }^{\circ} 1$, ci-dessous

Tableau n ${ }^{\circ}$. Matériels utilisés pour l'exploitation de l'or

\begin{tabular}{|l|l|l|}
\hline \multirow{4}{*}{$\begin{array}{l}\text { Village } \\
\text { d'enquête }\end{array}$} & \multicolumn{2}{|l|}{ Arsenal matériels en usage par les exploitants } \\
\cline { 2 - 3 } & Pour le creusage & $\begin{array}{l}\text { Pour la remontée de la } \\
\text { poussière }\end{array}$ \\
\cline { 2 - 3 } & burins, pioches, houes & Puisette \\
\cline { 2 - 3 } & $\begin{array}{l}\text { Proportion d'enquêtés en \% } \\
\text { se reconnaissant utilisateurs }\end{array}$ & $\begin{array}{l}\text { Proportion d'enquêtés en \% } \\
\text { se reconnaissant utilisateurs }\end{array}$ \\
\hline Mandièla & 100 & 100 \\
\hline Monpièla & 100 & 100 \\
\hline Nampala & 100 & 100 \\
\hline Tièkourala & 100 & 100 \\
\hline Tonokala & 100 & 100 \\
\hline Tofola & 100 & 100 \\
\hline
\end{tabular}

Source : Enquêtes de terrain, 2019

L'état des matériels révélé par le Tableau $\mathrm{n}^{\circ} 1$ atteste le caractère d'orpaillage traditionnel de l'activité d'exploitation minière pratiquée par les populations dans le Ganadougou. Au moyen desdits matériels les orpailleurs ont ouvert plusieurs sites dans le paysage de différents villages. Les puits qui y sont réalisés présentent des différences les uns avec les autres.

- «Woyo » : c'est une sorte de puits creusé sur une très grande profondeur. Il peut atteindre les $60 \mathrm{~m}$. Les orpailleurs le réalisent en faisant office de véritables ingénieurs. Leur ingénierie s'adresse à trois caractéristiques du puits. La première est la réalisation de véritables marches d'escalier facilitant les descentes et les remontées. A cet effet, le woyo ne garde pas la forme verticale des puits ordinaires. La deuxième est la prévision et la réalisation de poteaux de soutien du puits. La troisième porte sur le renforcement des poteaux par l'introduction des étais dans le puits.

- « Narakambo » : c'est une sorte de puits creusé sur une profondeur de $5 \mathrm{~m}$ au maximum. Il est aussi appelé 'Nara'. Cette appellation très courante est celle adoptée pour la présente recherche.

- «Zambougou»: c'est une sorte de puits non profond. Pour estimer sa profondeur les orpailleurs font référence à des mesures pratiques. Il s'agit de leur genou ou de leur hanche. Ainsi, ils s'expriment en disant que tel Zambougou fait une hanche et tel autre fait un genou. 
-Pi : il est très rare. Ce type de site est creusé sous forme de bassin d'eau sur les anciens sites ou déjà exploités.

Si les puits sont, par leur appellation et leur profondeur, différents les uns des autres, il est important de signaler l'existence de leur rapprochement à travers certains points communs, voir le Tableau $\mathrm{n}^{\circ} 2$, ci-dessous.

Tableau $n^{\circ} 2$. Nominations et conditions d'exploitation des puits sur les sites d'orpaillage dans le Ganadougou

\begin{tabular}{|c|c|c|c|}
\hline \multirow{3}{*}{$\begin{array}{l}\text { Village } \\
\text { d'enquête }\end{array}$} & $\begin{array}{l}\text { Appellation des puits } \\
\text { sur les sites }\end{array}$ & \begin{tabular}{|l|} 
Conditions \\
d'accès
\end{tabular} & Exploitant de l'or \\
\hline & \begin{tabular}{|ll} 
Woyo, & Nara, \\
Zambougou & \\
\end{tabular} & \begin{tabular}{|l|}
$\begin{array}{l}\text { Payantes pour } \\
\text { tous }\end{array}$ \\
\end{tabular} & $\begin{array}{l}\text { Aucune distinction entre } \\
\text { autochtone et allochtone }\end{array}$ \\
\hline & $\begin{array}{llr}\text { Proportion en } \% & \text { des } \\
\text { exploitants } & & \text { se } \\
\text { reconnaissant } & & \\
\text { utilisateurs } & \text { des } \\
\text { appellations } & & \\
\end{array}$ & $\begin{array}{l}\text { Proportion des } \\
\text { exploitants en \% } \\
\text { ayant accédé aux } \\
\text { puits par voie de } \\
\text { paiement }\end{array}$ & $\begin{array}{l}\text { Proportion des } \\
\text { exploitants en \% n'avoir } \\
\text { constaté une différence } \\
\text { de traitement en fonction } \\
\text { des origines }\end{array}$ \\
\hline Mandièla & 100 & 100 & 100 \\
\hline Monpièla & 100 & 100 & 100 \\
\hline Nampala & 100 & 100 & 100 \\
\hline Tièkourala & 100 & 100 & 100 \\
\hline Tonokala & 100 & 100 & 100 \\
\hline Tofola & 100 & 100 & 100 \\
\hline
\end{tabular}

Source : Enquête de terrain, 2019

Pour un géographe la lecture du tableau permet de retenir un fait très intéressant. Fondamentalement les puits ne singularisent aucun village. Ils sont donc facteur d'uniformisation des villages sites d'orpaillage. Relativement au point de vue d'un économiste le caractère payant de l'accès constitue un fait majeur à retenir, la rente foncière. Ladite rente $\mathrm{a}$, en milieu rural, des retombées bien connues depuis très longtemps. Les fondements mêmes d'une société locale sont remis en cause Jollivet (1978, p.7). Ne constituant pas l'objet de la présente recherche, l'effet de la rente foncière sur le fondement du système économique des populations du Ganadougou est très intéressant à aborder à l'avenir.

\section{Le niveau d'ancrage de l'activité}

Dans une acception large du mot, Raulet-Crozet (2008) assimile à un ancrage toute situation de gestion inscrite localement et affectant un collectif d'acteurs F. Bousquet (2014, p.68). Une telle approche nous parait très pertinente dans le cadre de notre recherche. En effet, l'orpaillage relève de la gestion des instances locales et affecte le collectif des orpailleurs. L'intérêt pour nous d'aborder l'ancrage de l'activité d'orpaillage est de cerner son importance dans l'espace du Ganadougou. Aussi, est-il nécessaire de retenir deux faits fondamentaux. 
-La distribution des sites, paramètre de lecture de l'assise territoriale. La carte $\mathrm{n}^{\circ} 2$, ci-dessous, donne des indications.

Comme l'indique la carte, $40 \%$ des communes soit quatre (4) sur dix (10) sont directement des entités administratives impliquées par l'orpaillage. Territorialement le fait est majeur. En effet, les quatre communes représentent $1605 \mathrm{~km}^{2}$ soit $51 \%$ de la totalité de l'espace du Ganadougou. En termes de nombre de villages lesdites communes totalisent 75 soit $59 \%$ des villages. Ils sont occupés par 53\% soit 51160 habitants selon les chiffres disponibles du recensement de 2009.

Du point de vue géographique la distribution des sites d'orpaillage est créatrice de réels contrastes spatiaux. Globalement un nord vide s'oppose à un sud pourvu en sites. En changeant d'échelle pour s'intéresser de très près au sud pourvu, des différences notoires apparaissent également. Dans un premier temps, à l'absence totale de site dans certaines communes du sud est opposable leur présence dans d'autres. Dans un second temps, les communes pourvues de sites n'échappent pas à une hiérarchie fondée sur la différence des nombres de sites. Douze sites sont présents dans la seule commune de Nièna contre seulement deux à Zanièna ; quatre à Finkolo Ganadougou ; un à N'tjikouna. Au total le Ganadougou compte dix neufs (19) sites.

Les différentes situations de contrastes constatés sont, à l'échelle globale comme à l'échelle du sud pris singulièrement, des indices pertinents porteurs de multiples enjeux. Ils relèvent du champ des géographes tout comme des économistes. Ce sont les questions de type de relation entre localités pourvues et celles dépourvues de sites, les formes de mutations spatiales et socio-économiques. Très intéressantes elles sont abordées dans les articulations suivantes de la présente recherche. 


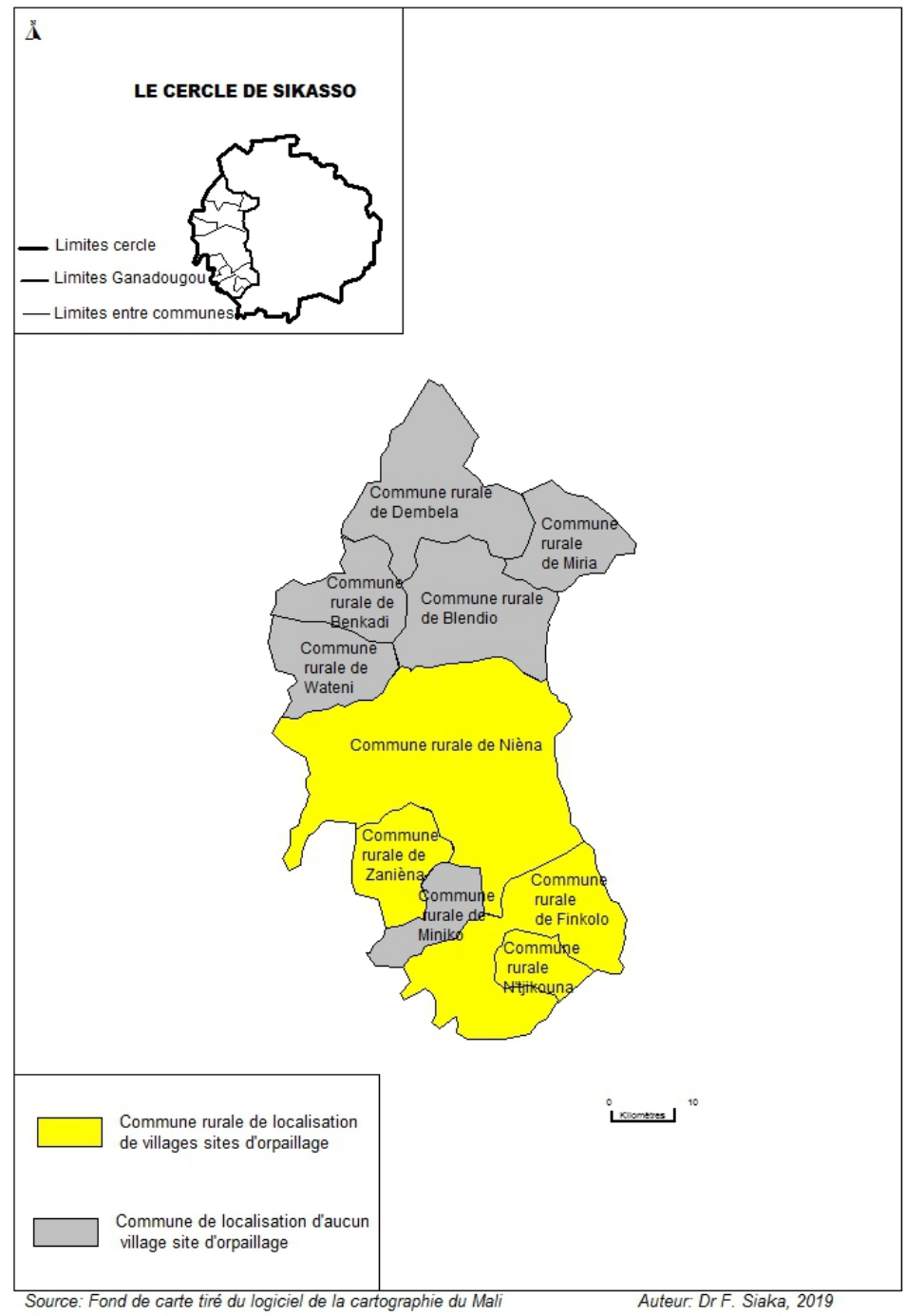

Carte ${ }^{\circ}{ }^{2}$. Ganadougou : distribution spatiale des sites d'orpaillage

-l'adhésion des populations est l'élément pertinent de compréhension du poids d'une activité dans le système économique de toute localité. Qu'en-est-il pour l'orpaillage dans le Ganadougou ? Le Tableau $\mathrm{n}^{\circ} 3$, ci-dessous, donne des orientations.

Le Tableau $\mathrm{n}^{\circ} 3$ révèle des informations très intéressantes lisibles à différents niveaux. Le premier se rapporte à l'échelle globale de l'espace du Ganadougou. Il ressort la généralisation de l'implication des populations dans la pratique de l'activité. D'une part, il prévaut une très forte participation des populations dans les villages sites.

A leur niveau au moins $52 \%$ des familles sont concernées. D'autre part, $12 \%$ des familles des villages non sites ont un membre de leur famille dans l'orpaillage. Cela met la lumière sur l'existence de relation entre les 
villages sites et ceux non sites. S'inscrivant dans le registre du phénomène des migrations de travail ladite relation présente divers enjeux.

Ces lieux de «ruée vers l'or» sont caractérisés par un afflux important d'orpailleurs étrangers dont les règles et principes s'avèrent souvent incompatibles avec ceux des populations locales, engendrant une situation de pluralisme normatif. Au départ, tous ces sites ont connu une période d'appropriation, parfois violente, des ressources et de fortes tensions au sein des groupes migrants (Grätz, 2004, p. 140).

Le second niveau de lecture est l'opposition entre les formes de participation des deux catégories de villages. A l'échelle des villages sites la pratique de l'orpaillage ne tient nullement compte du sexe. Les familles font participer leurs membres de sexe masculin comme ceux de sexe féminin. Cela n'est pas le cas des villages non sites. A leur niveau les familles excluent de la participation à l'orpaillage leur membre de sexe féminin.

A la lumière des développements faits, supra, sur les deux éléments de l'ancrage retenus, il apparait que l'orpaillage fait figure d'activité très importante dans le système économique des populations du Ganadougou. Ainsi, jouissant de statut d'activité à notoriété avérée, quel dispositif de sa gouvernance les populations ont-elles mis en place ?

\begin{tabular}{|c|c|c|c|c|c|c|c|c|c|}
\hline \multirow{2}{*}{$\begin{array}{l}\text { Nombre } \\
\text { de } \\
\text { membres } \\
\text { de la } \\
\text { famille de } \\
\text { sexe } \\
\text { masculin } \\
\text { pratiquant } \\
\text { l'orpaillage }\end{array}$} & \multicolumn{4}{|c|}{$\begin{array}{c}\text { Nombre de famille se déclarant dans le cas par } \\
\text { type de village }\end{array}$} & \multirow{2}{*}{$\begin{array}{c}\text { Nombre } \\
\text { de } \\
\text { membres } \\
\text { de la } \\
\text { famille de } \\
\text { sexe } \\
\text { féminin } \\
\text { pratiquant } \\
\text { l'orpaillage }\end{array}$} & \multicolumn{4}{|c|}{$\begin{array}{c}\text { Nombre de famille se déclarant dans le cas par } \\
\text { type de village }\end{array}$} \\
\hline & $\begin{array}{c}\text { Village } \\
\text { non site } \\
\text { d'orpaillage }\end{array}$ & $\begin{array}{l}\text { Proportion } \\
\text { en } \%\end{array}$ & $\begin{array}{l}\text { Village site } \\
\text { d'orpaillage }\end{array}$ & $\begin{array}{l}\text { Proportion } \\
\quad \text { en } \%\end{array}$ & & $\begin{array}{c}\text { Village } \\
\text { non site } \\
\text { d'orpaillage }\end{array}$ & $\begin{array}{l}\text { Proportion } \\
\text { en } \%\end{array}$ & $\begin{array}{l}\text { Village site } \\
\text { d'orpaillage }\end{array}$ & $\begin{array}{l}\text { Proportion } \\
\text { en } \%\end{array}$ \\
\hline 0 & 15 & 88 & 23 & 28 & 0 & 17 & 100 & 39 & 48 \\
\hline 1 & 1 & 6 & 14 & 17 & 1 & 0 & 0 & 12 & 15 \\
\hline 2 & 0 & 0 & 14 & 17 & 2 & 0 & 0 & 3 & 4 \\
\hline 3 & 1 & 6 & 10 & 12 & 3 & 0 & 0 & 7 & 9 \\
\hline 4 & 0 & 0 & 12 & 15 & 4 & 0 & 0 & 9 & 11 \\
\hline Total & 17 & 100 & 82 & 100 & Total & 17 & 100 & 81 & 100 \\
\hline
\end{tabular}

Tableau $n^{\circ} 3$. Les catégories de villages et la participation des populations à la pratique de l'activité d'orpaillage

\section{De l'individu a la Communaute Villageoise, Une Veritable Main Mise des Populations sur les Ressources Auriferes}

La main mise sur une ressource est le contrôle de l'accès, de l'exploitation et la gestion de ladite ressource. En tant que telle la main mise implique la notion de statut de la ressource déterminé par le régime foncier. Il 
n'est pas à comprendre exclusivement comme un ensemble de règles et de procédures de prise de décision explicites (régime légal), mais également la traduction explicite ou implicite de principes et de normes précis (Saël GagnéOuellet, 2013). En la matière, il prévaut au Mali un pluralisme juridique dans le cadre des activités minières (Belem, 2010 cité par Gagne-Ouellet). Ledit pluralisme est décrié par les chercheurs du Groupe d'étude et de recherche en sociologie et droit appliqué (GERSDA). En effet, les conventions d'établissement qui définissent les modalités d'exploitation, sont négociées entre le gouvernement national et les sociétés minières sans la participation des communautés locales (Keita et al., 2008).

Dans le paysage minier du Ganadougou sont absentes les sociétés minières. En rapport avec de tel contexte la main mise doit s'analyser à travers les rôles clés. Le Tableau $\mathrm{n}^{\circ} 4$, ci-dessous, donne la situation des différents acteurs et leurs rôles.

Tableau $\mathbf{n}^{\circ}$ 4. Acteurs et leurs rôles

\begin{tabular}{|c|l|l|l|l|}
\hline & $\begin{array}{l}\text { Rôle du villageois } \\
\text { reconnu } \\
\text { propriétaire de terre }\end{array}$ & $\begin{array}{l}\text { Rôle chef du de } \\
\text { village }\end{array}$ & $\begin{array}{l}\text { Rôle du } \\
\text { 'ton' } \\
\text { villageois }\end{array}$ & Rôle Maire \\
\cline { 2 - 5 } d'enquête & Autorisation & $\begin{array}{l}\text { Information } \\
\text { des tons }\end{array}$ & Sécurité & Sensibilisation \\
\cline { 2 - 5 } & $\begin{array}{l}\text { Proportion en \% } \\
\text { d'enquêtés } \\
\text { reconnaissant le } \\
\text { rôle }\end{array}$ & $\begin{array}{l}\text { Proportion en } \\
\text { \% d'enquêtés } \\
\text { reconnaissant } \\
\text { le rôle }\end{array}$ & $\begin{array}{l}\text { Proportion } \\
\text { en \% } \\
\text { d'enquêtés } \\
\text { reconnaissant } \\
\text { le rôle }\end{array}$ & $\begin{array}{l}\text { Proportion en } \\
\text { \% d'enquêtés } \\
\text { reconnaissant } \\
\text { le rôle }\end{array}$ \\
\hline Mandièla & 100 & 100 & 100 & 100 \\
\hline Monpièla & 100 & 100 & 100 & 100 \\
\hline Nampala & 100 & 100 & 100 & 100 \\
\hline Tièkourala & 100 & 100 & 100 & Oui \\
\hline Tonokala & 100 & 100 & 100 & 100 \\
\hline Tofola & 100 & 100 & 100 & 100 \\
\hline
\end{tabular}

Source : Enquêtes de terrain, 2019

Comme l'indique le tableau, le propriétaire terrien est le maître du jeu. En amont il autorise le chef de village à déclencher l'opération d'exploitation. Ce dernier fait une implication de la communauté à travers les tons (groupement). En effet, les tons se composent d'un "tonboloma" (membre du groupement) et d'un "dozo" (chasseur) qui sont les deux représentants mandatés par chacun des villages frontaliers au village siège du site. Corrélativement à leur activité et à leur statut les fonds engendrés par les attributions de puits sont répartis en deux : une première part pour le propriétaire ; une deuxième part revenant au chef de village et aux tons.

La mairie, maintenue dans un rôle quelconque de sensibilisation, participe très peu. Or, la mairie, suivant les textes de la décentralisation, a un autre rôle. Elle doit procéder à la délimitation de l'espace à exploiter. A ce 
titre et sur la base de tarif standard fixé par autorités locales coutumières, chaque puits exploité doit lui rapporter 1000f. Ne le faisant pas, la tenue des premiers rôles par les communautés villageoises à travers leurs représentants est significative de leur main mise sur leur ressource. Elle correspond à une réelle opérationnalisation de forme galvaudée de la politique de la décentralisation. Son origine remonte aux premières heures du renouveau démocratique. En effet, Le mouvement insurrectionnel de mars 1991 fut à son tour porteur d'un nouveau message relatif au bè jè fanga (littéralement : le pouvoir populaire), qui réclamait le partage égalitaire du pouvoir entre tous les citoyens. La dérive anarchique que cela engendra, et qui affaiblit considérablement les fondements de l'Etat, amena celui-ci à restaurer péniblement son autorité sérieusement entamée B. Kassibo (2006, p 8). Néanmoins, la main mise des populations sur leur ressource soulève des questionnements de fond. Ils sont relatifs à deux préoccupations majeures. Il s'agit du statut des ressources naturelles dans un espace communal; des modalités d'exploitation des ressources dans le sens de l'intérêt du développement global de la commune.

En ne percevant pas les préoccupations susmentionnées, les autorités communales se livrent à deux types de clientélismes. Le premier est électoraliste. A cet effet, pour garder les électeurs et de pouvoir se maintenir longtemps aux affaires, les autorités municipales se tiennent dans le mutisme et laissent les populations disposer des ressources comme bon leur semble. Le deuxième est financier. En effet, à chaque opération d'attribution des puits aux exploitants, un certain nombre de puits sont donnés aux agents de la mairie, de la gendarmerie et du bureau du sous-préfet si présents. Il en résulte, avec diverses traductions, une véritable fragilisation de l'autorité des responsables du développement communal. La difficulté fondamentale est liée à la crise de collaboration. Consécutivement la mairie se trouve dans l'impossibilité d'asseoir son autorité. Cela en dépit de son statut d'autorité compétente officiellement investie. L'encadré suivant en est la parfaite confirmation du problème d'autorité de la mairie.

\section{Encadré}

Le maire est impuissant face à l'ouverture et à la fermeture. La raison est qu'il n'est nullement associé dès le départ. Aussi, le recouvrement des taxes est un véritable problème. Souvent il arrive que les villageois s'interposent entre l'agent de recouvrement et les commerçants sur les sites. Il y a également l'opacité entretenue. Certains sites deviennent opérationnels sans que le maire en soit informé. Les chefs de villages n'apportent pas les vrais renseignements ou n'en apportent pas du tout. Du coup, la quote-part devant lui revenir n'est pas perçue, ou à moitié et jamais intégralement.

Finkolo, 15 avril 2019

Le Secrétaire général de la maire mandaté par le Maire à nous recevoir 
Le tableau de la gouvernance des autorités coutumières, abordée supra, soulève une interrogation essentielle. Quel développement procure-t-elle aux populations?

\section{Les Retombees de la Gouvernance de l'orpaillge Pour les Populations Villageoises}

Elles s'adressent aux conséquences qui peuvent être très multiples et diverses. Dans le cadre de la présente recherche l'intérêt porte exclusivement sur certains aspects que sont le développement économique et celui social.

Les capacités de mobilisation des moyens de production agricole, éléments traducteurs des potentialités d'investissements économiques des producteurs

Elles sont des éléments pertinents suffisamment expressifs des niveaux de pouvoir d'achat des populations dans les différentes catégories de villages de l'espace du Ganadougou. 
Tableau $\mathbf{n}^{\circ}$ 5. Les investissements dans la production agricole pendant la campagne de 2019

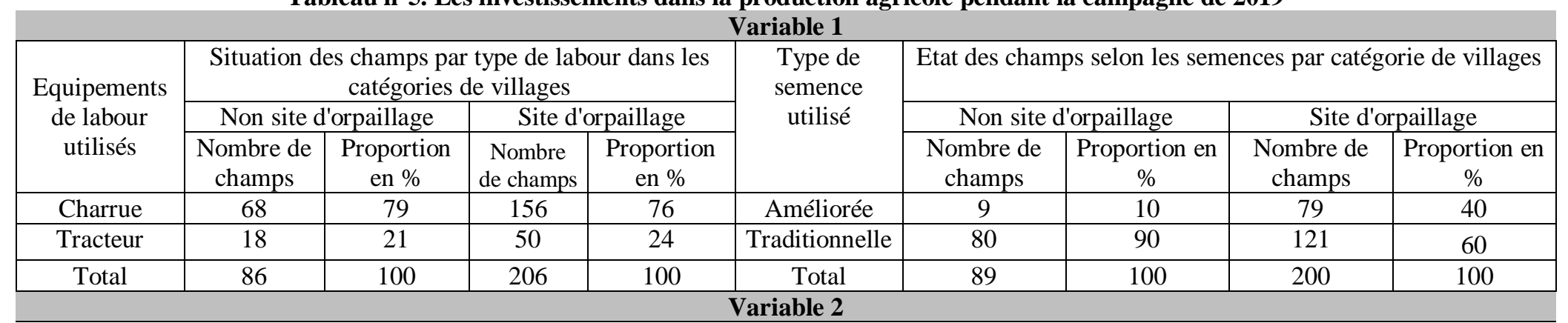

Situation d'utilisation de l'engrais par les populations dans les catégories de villages

\begin{tabular}{|c|c|c|c|c|c|c|c|c|c|}
\hline \multirow[b]{3}{*}{ Effectif sondé } & \multicolumn{4}{|c|}{ Non site d'orpaillage } & \multirow[b]{3}{*}{ Effectif sondé } & \multicolumn{4}{|c|}{ Site d'orpaillage } \\
\hline & \multicolumn{4}{|c|}{ Sac de $50 \mathrm{~kg}$} & & \multicolumn{4}{|c|}{ Sac de $50 \mathrm{~kg}$} \\
\hline & $\begin{array}{l}\text { Nombre } \\
\text { utilisé } \\
\text { déclaré }\end{array}$ & $\begin{array}{c}\text { Prix } \\
\text { unitaire }\end{array}$ & $\begin{array}{c}\text { Montant } \\
\text { total } \\
\text { investi }\end{array}$ & $\begin{array}{l}\text { Montant } \\
\text { moyen } \\
\text { sensé } \\
\text { investi par } \\
\text { sondé }\end{array}$ & & \begin{tabular}{|c|} 
Nombre \\
utilisé déclaré
\end{tabular} & Prix unitaire & $\begin{array}{l}\text { Montant total } \\
\text { investi }\end{array}$ & $\begin{array}{l}\text { Montant } \\
\text { moyen sensé } \\
\text { investi par } \\
\text { sondé }\end{array}$ \\
\hline 27 & 636 & 13000 & 8268000 & 306222 & 72 & 2600 & 13000 & 33800000 & 469444 \\
\hline
\end{tabular}

\section{Variable 3}

Utilisation des herbicides dans les catégories de villages

\begin{tabular}{|c|c|c|c|c|c|c|c|c|c|}
\hline \multicolumn{10}{|c|}{ Utilisation des herbicides dans les catégories de villages } \\
\hline \multicolumn{5}{|c|}{ Non site d'orpaillage } & \multicolumn{5}{|c|}{ Site d'orpaillage } \\
\hline \multirow[b]{2}{*}{ Effectif sondé } & \multicolumn{4}{|c|}{ Bidon d'herbicide } & \multirow[b]{2}{*}{ Effectif sondé } & \multicolumn{4}{|c|}{ Bidon d'herbicide } \\
\hline & $\begin{array}{l}\text { Nombre } \\
\text { utilisé } \\
\text { déclaré }\end{array}$ & $\begin{array}{c}\text { Prix } \\
\text { unitaire }\end{array}$ & $\begin{array}{c}\text { Montant } \\
\text { total } \\
\text { investi }\end{array}$ & $\begin{array}{l}\text { Montant } \\
\text { moyen } \\
\text { sensé } \\
\text { investi par } \\
\text { sondé }\end{array}$ & & $\begin{array}{c}\text { Nombre } \\
\text { utilisé déclaré }\end{array}$ & Prix unitaire & $\begin{array}{l}\text { Montant total } \\
\text { investi }\end{array}$ & $\begin{array}{l}\text { Montant } \\
\text { moyen sensé } \\
\text { investi par } \\
\text { sondé }\end{array}$ \\
\hline 26 & 501 & 4500 & 2254500 & 86712 & 70 & 2416 & 4500 & 10872000 & 155314 \\
\hline
\end{tabular}

Source : Enquête de terrain, 2019 
Le Tableau $\mathrm{n}^{\circ} 5$ conduit à quelques intéressantes constatations. Elles sont relatives à l'exceptionnel premier rang des villages non sites d'orpaillage dans l'usage de la charrue d'une part, d'autre part celui de meneur des villages sites d'orpaillage dans l'usage des autres moyens de production.

Certes amplificatrice des capacités de mise en valeur des terres à travers la force animale, l'important usage de la charrue n'est plus un atout. En effet, l'analyse de la pluviométrie mensuelle fait apparaittre une répartition aléatoire de la pluviosité en début de la saison des pluies (DNM) (2007, p.25). Ce contexte climatique est responsable d'une tendance d'établissement de forte corrélation entre réussite des cultures et labour à partir des premières pluies. L'arrivée desdites premières pluies coïncidant avec la période d'indisponibilité des bœufs de labour, la politique étatique est l'encouragement de l'usage des tracteurs. Dans le même registre de solutions aux problèmes de l'agriculture, la Direction Nationale de la Météorologie (DNM), chargée de piloter le Programme d'Action National d'Adaptation aux changements climatiques PANA, préconise le développement des variétés améliorées et adaptées des principales cultures céréalières à la sécheresse.

La situation des modalités, usage assez important des tracteurs et très important des semences améliorées, est un critère suffisant attestant le développement de l'activité de production agricole des villages sites d'orpaillage. Elle est techniquement en phase avec les exigences climatiques du milieu. En plus des dispositions de gestion des contraintes climatiques, les producteurs entretiennent des stratégies de réalisation des grandes productions à travers l'usage important des engrais et herbicides. La faible observation des mêmes conditions par les producteurs des villages non sites d'orpaillage s'explique par leur manque de moyen et leur faible capacité d'endettement. En effet, ils ont le coton dans leur système de production. Par le biais de cette culture ils bénéficient des intrants sous forme de crédit agricole. A l'opposé, suivant les résultats de nos enquêtes il ressort qu'à l'échelle des villages sites les familles reçoivent, de manière permanente, les appuis financiers de leurs membres impliqués dans l'orpaillage. En plus des crédits agricoles les familles ont des ressources financières additionnelles. Du coup, les villages sites d'orpaillage se constituent en véritable bastion agricole du Ganadougou. Le Tableau $\mathrm{n}^{\circ} 6$, ci-dessous, en constitue un argument supplémentaire à travers la structuration du paysage agraire. 
Tableau n ${ }^{\circ}$ 6. Structure du paysage agraire dans les catégories de villages

\begin{tabular}{|c|c|c|c|c|c|}
\hline \multirow[b]{2}{*}{$\begin{array}{l}\text { Catégorie } \\
\text { de champ }\end{array}$} & \multirow[b]{2}{*}{$\begin{array}{c}\text { Classe de } \\
\text { superficie } \\
\text { en ha }\end{array}$} & \multicolumn{2}{|c|}{ Non site d'orpaillage } & \multicolumn{2}{|c|}{ Site d'orpaillage } \\
\hline & & $\begin{array}{l}\text { Superficie } \\
\text { totale par } \\
\text { catégorie }\end{array}$ & $\begin{array}{l}\text { Proportion } \\
\text { en } \%\end{array}$ & $\begin{array}{l}\text { Superficie } \\
\text { totale par } \\
\text { catégorie }\end{array}$ & $\begin{array}{l}\text { Proportion } \\
\text { en } \%\end{array}$ \\
\hline Petit & {$[0-4[$} & 65 & 77 & 193 & 84 \\
\hline Moyen & [4-8[ & 28 & 18 & 74 & 11 \\
\hline Grand & {$[8-12[$} & 24 & 5 & 47 & 4 \\
\hline Très grand & $>=12-<16$ & 0 & 0 & 66 & 2 \\
\hline Total & & 117 & 100 & 380 & 100 \\
\hline
\end{tabular}

Source : Enquêtes de terrain, 2019

Comme l'indique le Tableau ${ }^{\circ} 6$, le paysage agraire des villages sites d'orpaillage est à l'image d'une agriculture plus dynamique. On y trouve des champs dont la catégorie est totalement absente du paysage agraire des villages non sites d'orpaillage. Il s'agit des très grands champs dont le labour est exclusivement du ressort des tracteurs. Sur la base de nos enquêtes il existe des champs de 15 ha. Leurs tenants sont propriétaires de tracteurs contrairement à beaucoup d'autres producteurs dont l'usage des tracteurs est à titre locatif. 


\section{Le niveau d'accès des populations à certaines commodités de la vie courante, fait traducteur de développement} social dans les catégories de villages

Tableau de $n^{\circ}$. Situation des catégories de villages dans la satisfaction des besoins de la vie courante

\begin{tabular}{|c|c|c|c|c|c|c|}
\hline \multicolumn{7}{|c|}{ Variable 1} \\
\hline \multirow{3}{*}{$\begin{array}{l}\text { Source de l'eau } \\
\text { domestique }\end{array}$} & \multicolumn{6}{|c|}{ Accès à l'eau consommée par les familles dans les catégories de villages } \\
\hline & \multicolumn{3}{|c|}{ Non site d'orpaillage } & \multicolumn{3}{|c|}{ Site d'orpaillage } \\
\hline & $\begin{array}{l}\text { Effectif famille } \\
\text { sondée }\end{array}$ & $\begin{array}{c}\text { Nombre de famille } \\
\text { utilisatrice }\end{array}$ & $\begin{array}{c}\text { Proportion famille } \\
\text { utilisatrice en } \%\end{array}$ & $\begin{array}{l}\text { Effectif famille } \\
\text { sondée }\end{array}$ & $\begin{array}{c}\text { Nombre de famille } \\
\text { utilisatrice }\end{array}$ & $\begin{array}{l}\text { Proportion famille } \\
\text { utilisatrice en } \% \text { de }\end{array}$ \\
\hline $\begin{array}{l}\text { Pompe à motricité } \\
\text { humaine }\end{array}$ & \multirow{4}{*}{27} & 17 & 63 & \multirow[t]{4}{*}{ 年 } & 56 & 78 \\
\hline Puits à grand diamètre & & 3 & 11 & & 9 & 13 \\
\hline Puits traditionnel & & 7 & 26 & & 6 & 8 \\
\hline Robinet personnel & & 0 & 0 & & 1 & 1 \\
\hline Total & & 27 & 100 & & 72 & 100 \\
\hline \multicolumn{7}{|c|}{ Variable 2} \\
\hline \multirow{3}{*}{$\begin{array}{l}\text { Les différents éléments } \\
\text { relatifs aux besoins }\end{array}$} & \multicolumn{6}{|c|}{ Les réalités d'équipement et d'alimentation des popu } \\
\hline & \multicolumn{3}{|c|}{ Non site d'orpaillage } & \multicolumn{3}{|c|}{ Site d'orpaillage } \\
\hline & $\begin{array}{l}\text { Effectif famille } \\
\text { sondée }\end{array}$ & $\begin{array}{l}\text { Nombre de famille } \\
\text { concernée }\end{array}$ & $\begin{array}{l}\text { Proportion famille } \\
\text { concernée en \% }\end{array}$ & $\begin{array}{l}\text { Effectif famille } \\
\text { sondée }\end{array}$ & $\begin{array}{l}\text { Nombre de famille } \\
\text { concernée }\end{array}$ & $\begin{array}{l}\text { Proportion famille } \\
\text { concernée en } \% \text { de }\end{array}$ \\
\hline \multicolumn{7}{|c|}{ Déplacement } \\
\hline Moto personnelle & \multirow{2}{*}{27} & 22 & 81 & \multirow{2}{*}{72} & 70 & 97 \\
\hline Vélo personnel & & 5 & 19 & & 2 & 3 \\
\hline \multicolumn{7}{|c|}{ Eclairage de maison } \\
\hline Panneau solaire & \multirow{2}{*}{27} & 21 & 78 & \multirow{2}{*}{72} & 69 & 96 \\
\hline Lampe à pile & & 6 & 22 & & 3 & 4 \\
\hline \multicolumn{7}{|c|}{ Nature de la maison } \\
\hline Banco et en dur & \multirow{3}{*}{27} & 1 & 4 & \multirow{3}{*}{72} & 33 & 46 \\
\hline Tout en banco & & 26 & 96 & & 37 & 51 \\
\hline Tout en dur & & 0 & 0 & & 2 & 3 \\
\hline \multicolumn{7}{|c|}{ Couverture des besoins alimentaires } \\
\hline Acheté en partie & \multirow{3}{*}{27} & 15 & 56 & \multirow{3}{*}{72} & 31 & 43 \\
\hline Totalement acheté & & 0 & 0 & & 1 & 1 \\
\hline Totalement produit & & 12 & 44 & & 36 & 56 \\
\hline
\end{tabular}


La lecture du Tableau $\mathrm{n}^{\circ} 7$ révèle des niveaux différents de satisfaction des besoins de la vie courante. Ils sont en faveur des villages sites d'orpaillage. Cependant, certains faits majeurs sont à retenir. Il s'agit de la nature de la maison et de la couverture des besoins alimentaires. Relativement à la nature de la maison le style en dur est un indicateur de véritable mutation du paysage rural dans le Ganadougou. En effet, la caractéristique de la maison rurale est sa réalisation avec les matériaux locaux. Or, le ciment et le fer, principaux matériaux des maisons en dur, sont importés et exclusivement accessibles à partir de leur achat. C'est dire que la maison en dur est l'expression d'un niveau de vie nettement au dessus de la moyenne. A cet effet, elle est l'apanage des seuls privilégiés et procure la grande fierté à toutes les familles qui en disposent. En tant que telle la maison en dur est source de différenciation sociale. La différenciation spatiale est aussi une réalité à travers le caractère important de leurs proportions dans les villages sites d'orpaillage. Lesdites proportions ont contribué au développement d'une mentalité au niveau des populations. De manière unanime les villages sites d'orpaillage sont appelés les localités nanties du Ganadougou.

Le deuxième fait portant sur la couverture des besoins alimentaires est traducteur d'un profond malaise social dans les villages non sites d'orpaillage. Très majoritairement les populations achètent une partie de leurs besoins alimentaires. Symptomatique du caractère moins performant d'une agriculture sevrée d'investissement adéquat dans les moyens de production, comme l'indique le Tableau $\mathrm{n}^{\circ} 6$ supra, la proportion importante de population observant l'achat d'une partie des besoins met toute la lumière sur les problèmes alimentaires vécus dans les villages non sites d'orpaillage. Cela n'est pas une spécificité du Ganadougou. On identifie des poches d'insécurité alimentaire dans toutes les régions du pays, indiquant une vulnérabilité particulièrement importante au niveau des zones disposant de peu de moyen d'existence FEWS NET (2015, p.17). En termes de populations vulnérables, le Tableau n ${ }^{\circ} 8$, ci-dessous, donne des indications relatives aux communes non sites d'orpaillage dans le Ganadougou.

Tableau $n^{\circ}$ 8. Populations vulnérables par sexe dans les communes non sites d'orpaillage dans le Ganadougou

\begin{tabular}{|c|c|c|c|}
\hline \multirow{2}{*}{ Commune } & \multicolumn{2}{|c|}{ Populations vulnérables } & \multirow{2}{*}{ Total } \\
\cline { 2 - 3 } & Sexe masculin & Sexe féminin & \\
\hline Benkadi & 462 & 487 & 949 \\
\hline Blendio & 698 & 762 & 1460 \\
\hline Dembela & 731 & 759 & 1490 \\
\hline Miniko & 200 & 192 & 392 \\
\hline Miria & 554 & 636 & 1190 \\
\hline Wateni & 369 & 371 & 740 \\
\hline
\end{tabular}

Source : Cellule de planification et de statistique, 2014 


\section{Conclusion}

L'activité d'exploitation de l'or est une pratique des populations dans le Ganadougou. La recherche a poursuivi certains objectifs. Il s'agit de connaitre l'importance et la nature des pratiques d'exploitation de l'or ; de connaitre les modalités de la gouvernance des ressources foncières aurifères ; de faire une analyse de la traduction spatiale du développement lié à la gouvernance en place. Il ressort des résultats que la pratique d'exploitation, en référence aux indications du code minier malien, est de l'orpaillage traditionnel. Il est d'une importance notoire compte tenu de deux principales raisons. Ce sont l'étendue territoriale formée par les communes rurales directement impliquées et le niveau très élevé de l'adhésion des populations. Cela confirme la première hypothèse de recherche. Relativement à la gouvernance les populations villageoises sont pleinement les premiers responsables. Ce résultat confirme la deuxième hypothèse. Quant au développement engendré son analyse laisse comprendre sa dimension économique et celle sociale. Ainsi, la troisième hypothèse est confirmée.

En termes de traduction du développement l'orpaillage est facteur de fabrication de sous-espace très inégalitaire à l'échelle du Ganadougou. Profitant pleinement des filons de l'or les villages sites d'orpaillage se sont érigés en véritable pôle agricole caractérisé par une agriculture en phase avec les exigences climatiques et de grande productivité. Ladite agriculture s'oppose à celle des villages non sites d'orpaillage. Sur le plan social la même catégorisation spatiale prévaut. Si les villages sites d'orpaillage ont façonné un nouveau territoire avec un paysage de maison en dur digne de nantis, les villages non sites constituent un espace où prévaut une importante insécurité alimentaire.

\section{References:}

1. Bousquet, F. (2014). L'influence du lien personnel entre L'entrepreneur et le territoire sur l'ancrage territorial des PME, thèse de doctorat, Université de Bordeaux, 733 p.

2. Cellule de Planification et de Statistique (2015). Annuaire statistique 2014 du secteur du Développement rural, Ministère du Développement Rural, 147p.

3. Direction Nationale de La Meteorologie (2007). Programme d'Action National d'Adaptation aux Changements Climatiques, Ministère de l'équipement et des transports, $100 \mathrm{p}$.

4. Djire, M., Traore, K., Dembele, D., Dembele, A., Samassekou, M., \& Doumbo, M. (2008). Communautés locales et «manne aurifère » : les oubliées de la législation minière, iied, $41 \mathrm{p}$.

5. FEWS NET (2015). Enquête Nationale sur la Sécurité Alimentaire et Nutritionnelle (ENSAN) au Mali Juin 2015, FAO, 33 p. 
6. Grätz, T. (2004). Les frontières de l'orpaillage en Afrique occidentale, Autrepart $\mathrm{n}^{\circ} 30,16 \mathrm{p}$.

7. Jollivet, M. (1978). Rente foncière et gestion des ressources naturelles. Quelques remarques sur la rente foncière dans une perspective interdisciplinaire- In Etudes rurales, $\mathrm{n}^{\circ} 71-72$, 257-274 pp.

8. Kassibo, B. (2006). «La Décentralisation au Mali : État des Lieux », Bulletin $\mathrm{n}^{\circ} 14$ de l'APAD, $21 \mathrm{p}$.

9. Keita, S. (2001). Etude sur les mines artisanales et les exploitations minières à petite échelle, iied, Mining, Minerals and Sustainable Development, $\mathrm{N}^{\circ} 80,54 \mathrm{p}$.

10. Onudi, D. (2009). Atelier sous-régional d'information des pays de l'Afrique francophone sur les problèmes liés à l'orpaillage, rapport de synthèse, $34 \mathrm{pp}$.

11. Panella, C. (2007). L'éthique sociale du damansen, Éducation familiale et orpaillage artisanal dans le Basidibé (Wasolon, Mali), Cahiers d'études africaines, $23 \mathrm{p}$.

12. Saël Gagné, O. (2013). Révision du cadre réglementaire et réforme du régime minier malien : quel degré de rupture et de continuité?, Université du Québec à Montréal, Service des bibliothèques, $96 \mathrm{p}$.

13. Thune, M. (2011). L'industrialisation de l'exploitation de l'or à Kalsaka, Burkina Faso : une chance pour une population rurale pauvre ?, EchoGéo, 19 p.

14. WebNewsle360afrique.com (2019). Voici les 5 nouveaux plus grands producteurs d'or d'Afrique. Accessible sur URL : http://afrique.le360.ma/autres-pays/economie

15. WWW.Droit-Afrique.com: Mali code minier 2012, Loi n²012-015 du 27 février 2012. Accessible sur URL: http://www.droitafrique.com/upload/doc/mali/Mali-Code-2012-minier.pdf 